\title{
Arqueobotánica de Cueva Huenul 1 (Neuquén, Argentina): selección y procesamiento de especies vegetales
}

\author{
Carina Llano, Paula Sosa, Clara Sánchez Campoo y Ramiro Barberena \\ Recibido 20 de febrero 2019. Aceptado 10 de julio 2019
}

\begin{abstract}
RESUMEN
En este trabajo se presentan los últimos análisis arqueobotánicos del sitio Cueva Huenul 1, emplazado en el norte de la provincia del Neuquén (noroeste de Patagonia, Argentina). La secuencia cronológica del sitio abarca en forma discontinua desde la transición Pleistoceno-Holoceno hasta tiempos recientes. El objetivo es realizar un análisis diacrónico de las pautas de selección antrópica de especies y los patrones de procesamiento. Los resultados indican que los cazadores-recolectores utilizaron plantas disponibles localmente durante toda la secuencia ocupacional. Temporalmente, se observan cambios tanto a nivel de la diversidad de especies representadas como, en el caso específico del taxón Prosopis, en las pautas de procesamiento y consumo. Algunos taxones consumidos en el Holoceno tardío constituyen indicadores de estacionalidad y sugieren ocupaciones estivales en la cueva. Asimismo, se discuten datos sobre la presencia de especies domésticas en el registro, tales como Lagenaria sp. y Zea mays. Estos estudios se conectan con discusiones más amplias sobre la economía y organización espacial de las sociedades que habitaron el noroeste de Patagonia desde el poblamiento inicial.
\end{abstract}

Palabras clave: Arqueobotánica; Tendencias temporales; Patrones de procesamiento; Estacionalidad; Intercambio de especies domésticas.

\begin{abstract}
THE ARCHAEOBOTANY OF HUENUL CAVE 1 (NEUQUÉN, ARGENTINA): SELECTION AND PROCESSING OF VEGETABLE SPECIES. In this paper the most recent archaeobotanical results from the archaeological site Huenul Cave 1, located in northern Neuquén Province (northwestern Patagonia, Argentina), are presented. The site has a discontinuous temporal sequence extending from the Pleistocene-Holocene transition to recent times. The aim of the study is to develop a diachronic analysis of the human selection of taxa and processing patterns. The results indicate that hunter-gatherer societies systematically utilized locally available wild plants throughout the occupational sequence. Changes were recorded in the diversity of taxa consumed through time and, in the case of the taxon Prosopis, in the patterns of processing and use. Some of the taxa recovered for late Holocene layers constitute proxies of seasonality that suggest summer occupations at the site. Additionally, the presence of domesticated species such as Lagenaria sp. and Zea mays was assessed. This study contributes to the broader analysis of the economy and spatial organization of the human societies that have inhabited northwestern Patagonia since its initial peopling.
\end{abstract}

Keywords: Archaeobotany; Temporal trends; Plant processing; Seasonality; Exchange of domesticated taxa.

\footnotetext{
Carina Llano. Laboratorio de Paleoecología Humana (LPEH), Instituto Interdisciplinario de Ciencias Básicas (ICB), UNCUYO, CONICET, FCEN, Padre J. Contreras 1300 (5500) Mendoza, Argentina. E-mail: Ilano.carina@gmail.com

Paula Sosa. ICB, LPEH. Abel Zapata 274. Las Heras, Mendoza, Argentina. E-mail: psosamodon@gmail.com

Clara Sánchez. ICB, LPEH. Esmeralda 300. Cuadro Benegas, Mendoza, Argentina. E-mail: mclaracampoo@gmail.com

Ramiro Barberena. LPEH, ICB, UNCUYO, CONICET, FCEN. Las Cañas 1808 Guaymallén, Mendoza, Argentina. E-mail: ramidus28@gmail.com
} 


\section{INTRODUCCIÓN}

El registro arqueobotánico ofrece evidencia sólida de que la recolección de plantas cumplió un papel importante en la economía de las poblaciones cazadoras-recolectoras de Patagonia (Pérez de Micou 1991; Crivelli et al. 1996; Pérez y Erra 2011; Caruso 2012; Lema et al. 2012; Capparelli y Prates 2015; Belmar et al. 2017; Ciampagna y Mange 2017). Estos antecedentes en escala macrorregional suministran un valioso aporte para la interpretación local del registro de Cueva Huenul 1 ( $\mathrm{CH} 1$ de aquí en más).

El presente artículo muestra los hallazgos arqueobotánicos del sitio $\mathrm{CH} 1$, localizado en el norte de la provincia del Neuquén (Figura 1). Las primeras menciones sobre su existencia tuvieron lugar en relación con el arte rupestre en la década de 1970 (Fernández 1974-1975; Schobinger 1985; ver también Cordero et al. 2002). Posteriormente, se retomaron estos trabajos iniciales en el marco de un proyecto regional dirigido a caracterizar el uso humano del espacio en un paisaje heterogéneo a nivel topográfico y ecológico. En esta línea, este proyecto macro incluyó el análisis de conjuntos líticos, faunísticos, arqueobotánicos, de arte rupestre y paleoecología (Barberena et. al. 2015).
Este trabajo expande el análisis de la cuadrícula A1, excavada en el año 2009 (Llano y Barberena 2013), y presenta los resultados de las cuadrículas C1 y D1, excavadas en el año 2012. El objetivo general consiste en evaluar las prácticas de subsistencia desde el registro de macrorrestos vegetales, considerando sus implicaciones en términos de estacionalidad ocupacional del emplazamiento. Se tiene en cuenta la amplitud de dieta humana producto de la selección de taxones, por lo cual se evalúan su frecuencia, densidad y riqueza específica. Se enfoca particularmente en evaluar las tendencias temporales en las pautas de procesamiento y descarte antrópico de plantas. Para este análisis se cuenta con un robusto marco cronoestratigráfico para las cuadrículas A1-C1-D1, que incluye numerosas fechas taxón para las principales especies consumidas (ver abajo).

Las excelentes condiciones de preservación de los macrorrestos en toda la secuencia permiten reconocer indicadores tafonómicos de manejo y descarte antrópico. El análisis pone de relevancia la existencia de similitudes y diferencias en la composición taxonómica de los conjuntos, lo cual conlleva a una interpretación más compleja de las estrategias de aprovisionamiento, procesamiento y descarte de vegetales y, sobre esta base, de una reconstrucción

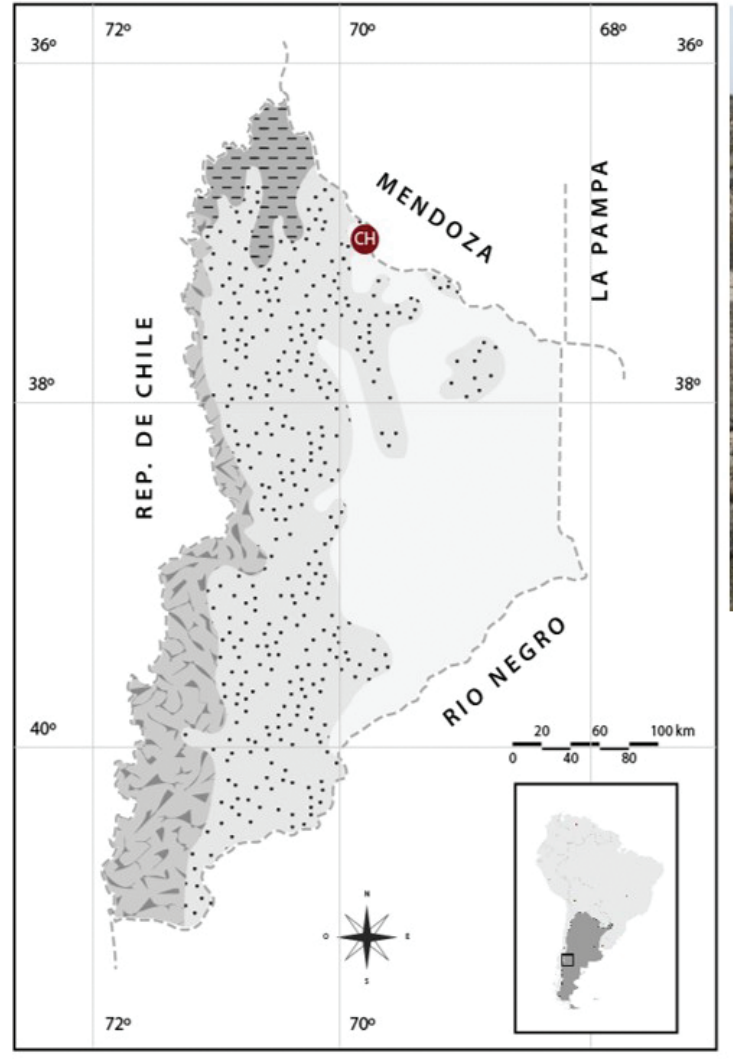

Altoandina Bosques Patagónicos Estepa Patagónica Monte

Figura 1. Localización del sitio arqueológico Cueva Huenul 1.
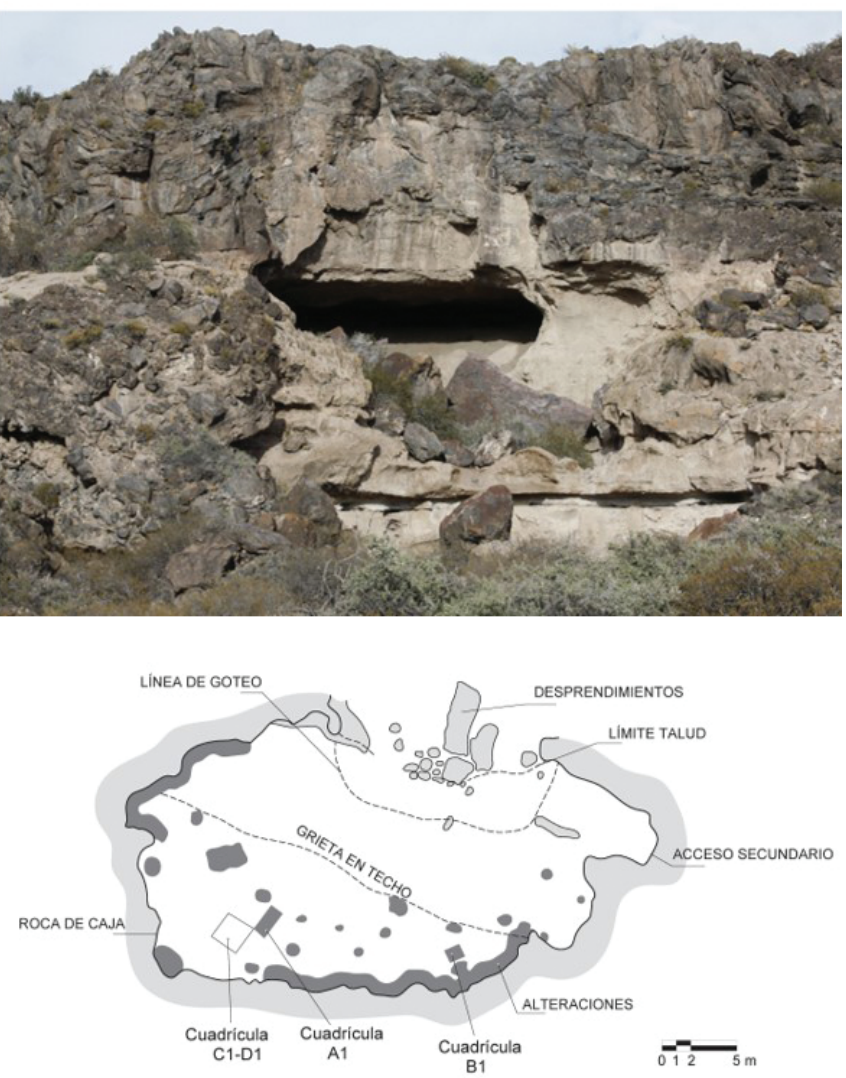
de la historia ocupacional del sitio mediante la integración con múltiples líneas de evidencia.

\section{$\mathrm{CH} 1$ en su contexto ecológico regional}

En la región sur de los Andes patagónicos (30$40^{\circ} \mathrm{S}$ ) ocurre la transición de los sistemas de circulación atmosférica Subtropical y Templado, lo que se traduce en un clima árido y semiárido (Páez et al. 2004). La cordillera occidental recibe precipitaciones promedio de $2000 \mathrm{~mm}$ anuales, mientras que las estepas patagónicas localizadas al este reciben menos de 300 mm anuales (Roig 1998). Estas condiciones climáticas crean como unidad fisiográfica un gradiente bioecológico pronunciado que genera ecosistemas variados en distancias cortas a partir del cual es posible distinguir tres regiones principales. De oeste a este, se localiza inicialmente la provincia fitogeográfica Altoandina (2600-2800 msnm), caracterizada por suelos rocosos muy pobres en materia orgánica, excepto en las turberas, que actúan como reservorios de agua donde la vegetación característica es una estepa de gramíneas y plantas en cojín. Los principales taxones son Azorella, Nassauvia, Maihueniopsis y Senecio. En segundo lugar se encuentra la provincia fitogeográfica Patagónica de llanuras, mesetas y montañas bajas dominantes con vegetación arbustiva y herbácea (Pappostipa, Mulinum, Chuquiraga), con una elevada capacidad de carga de herbívoros en la estación estival (Mendía 2006). Por último, la provincia fitogeográfica del Monte, compuesta por llanuras y bolsones con suelos en general arenosos con predominio de vegetación arbustiva xerófila (Larrea, Prosopis y Prosopidastrum) (Roig et al. 2009). Algunas de estas especies son clave desde el punto de vista de su potencial alimenticio para el consumo humano (Llano 2014).

\section{Historia paleoambiental}

El escenario paleoambiental del norte de Patagonia incluye el análisis de múltiples indicadores con diferencias en sensibilidad y resolución temporal (Markgraf et al. 2009; Fernández et al. 2012). Estos estudios se focalizan en el área de bosque y el ecotono estepa-monte (de Porras 2017).

En el tardiglacial ( 17.000 años cal. AP), las condiciones frías y secas se reflejan en el desarrollo de un bosque abierto de Nothofagus. Durante el Holoceno temprano (11.000-8000 años cal. AP), un aumento en la diversidad en los elementos de la estepa señalaría un incremento en la precipitación y temperatura, pero sin alcanzar los valores actuales. Para el Holoceno medio (8000-3000 años cal. AP), el registro demuestra condiciones húmedas, visualizado en la presencia de Cyperaceae. Finalmente, en el Holoceno tardío (últimos 3000 años cal. AP) se evidencian condiciones climáticas similares al presente, con precipitaciones invernales y veranos secos. Estas condiciones indican que el paisaje fitogeográfico se mantuvo sin alteraciones significativas en los últimos milenios (de Porras 2017).

En escala local, estudios preliminares del polen fósil de $\mathrm{CH} 1$ sugieren que las comunidades de arbustos y gramíneas estuvieron presentes en el entorno del sitio desde ca. 13.800 años cal. AP. Si bien los conjuntos polínicos no evidencian cambios sustantivos, hay diferencias en la composición. Entre ca. 13.800-9500, el polen indica la presencia de estepas graminosas típicas de la provincia Patagónica, integrada con arbustos (Lycium, Schinus). Estos resultados sugieren condiciones locales más frías que en el presente, consistentes con los resultados de microfauna de $\mathrm{CH} 1$ (Fernández et al. 2012). Para los últimos 1500 años, el polen indica la presencia de la transición Monte-Patagonia, con dominio de Lycium, Schinus y gramíneas en menor proporción, lo que refleja condiciones similares a la vegetación moderna (Pompei et al. 2012).

\section{MATERIALES Y MÉTODOS}

\section{Materiales}

El sitio $\mathrm{CH} 1$ está ubicado en la provincia del Monte, $8 \mathrm{~km}$ al este del ecotono con la provincia Patagónica (Figura 1). Consiste en una cueva originada por procesos erosivos cuyo espacio bajo reparo es de $30 \times 20 \mathrm{~m}$. El gran tamaño del espacio, sumado a la baja circulación de agua en un amplio sector del sitio, redundaron en excelentes condiciones de acumulación sedimentaria y preservación de materiales orgánicos. En total se excavaron cuatro cuadrículas. En este trabajo se presenta nueva información para las cuadrículas C1 y D1, que se integra con los datos ya publicados para la cuadrícula A1 (Llano y Barberena 2013). Cada una de estas tres tiene una dimensión de $2 \times 1 \mathrm{~m}$, lo que totaliza $6 \mathrm{~m}^{2}$ de superficie de excavación. No se presenta el análisis de la última cuadrícula excavada (B1) porque presenta procesos significativos de migración de materiales por remoción antrópica reciente. 
Considerando que la composición taxonómica de la cuadrícula B1 es semejante a las aquí estudiadas, se optó por excluirla del análisis detallado.

Para C1 y D1 se alcanzó una profundidad máxima de $1 \mathrm{~m}$ (4 $\mathrm{m}^{3}$ de volumen excavado). La excavación se realizó empleando niveles artificiales de extracción de $10 \mathrm{~cm}$ de potencia, numerados a partir de 1 desde la superficie hacia la base, y luego se efectuó la localización tridimensional de los hallazgos. La numeración de los componentes se estableció desde el más antiguo hasta el más reciente. Los sedimentos fueron tamizados en seco utilizando zarandas con malla de $1 \mathrm{~mm}$ de apertura. Todas las fechas mencionadas en el texto se presentan en años calibrados AP obtenidos con dos sigmas mediante la curva del hemisferio sur SHCAL 13 (Hogg et al. 2013). La secuencia cronoestratigráfica describe la sucesión en las tres cuadrículas analizadas (Tabla 1, detaIles en Barberena 2014).

Componente 1: extracciones artificiales 10 a 7. Las fechas disponibles abarcan un rango temporal de entre 16.600-13.600 años cal. AP. No se registraron evidencias arqueológicas in situ, aunque se destaca la notable abundancia de excrementos de carnívoros pequeños con una excelente preservación (Tietze et al. 2019). Se fecharon en forma directa dos coprolitos que corresponderían a perezoso. El límite superior de este componente está dado por una discordancia erosiva denominada Hiato 1.

Componente 2: extracciones artificiales 6 a 4 ubicadas inmediatamente por encima del Hiato 1. Incluye las primeras ocupaciones humanas en $\mathrm{CH} 1$ fechadas entre 11.30010.200 años cal. AP.

Componente 3: fue excavado como una unidad de extracción en sí misma. Corresponde a una estructura antrópica denominada $G$, un pozo relleno de material vegetal datado en 56005300 años cal. AP.

Componente 4: extracciones artificiales 3 a 1, fechadas entre 1500-300 años cal. AP. Coincide con un pulso de elevada ocupación regional.

\section{Métodos}

El planteo metodológico se basó en el análisis de macrorrestos (hojas, areolas, frutos, espinas, semillas) provenientes de muestras estratigráficas que reflejan el material disperso contenido en la matriz. La clasificación del material botánico se realizó a ojo desnudo y con microscopio estereoscópico (Zeiss Modelo Stemi DV4) con un aumento de 10x a 20x. Los materiales se encontraron secos en su totalidad. La identificación taxonómica se llevó a cabo por medio de anatomía comparada y observación de la morfología externa de los especímenes con la colección de referencia de frutos y semillas colectadas en las inmediaciones del sitio. Para ello se realizaron tres muestreos tomando puntos de intercepción (Mostacedo y Fredericksen 2000), en los que se recolectaron de manera sistemática los especímenes potencialmente explotables (resultados

\begin{tabular}{|c|c|c|c|c|c|c|}
\hline \multirow{2}{*}{ Procedencia } & \multirow{2}{*}{ Unidad } & \multirow{2}{*}{ Material } & \multirow{2}{*}{ Edad ${ }^{14} \mathrm{C}$} & \multirow{2}{*}{$\begin{array}{c}\text { Edad calibrada } \\
\text { AP }\end{array}$} & Mediana & \multirow{2}{*}{ Código } \\
\hline & & & & & (cal. AP) & \\
\hline \multicolumn{7}{|c|}{ Cuadrículas A1-C1 } \\
\hline \multirow{2}{*}{$\begin{array}{l}\text { A1. Nivel } 1 \\
(10-20 \mathrm{~cm})\end{array}$} & \multirow{2}{*}{1} & Lama guanicoe & \multirow{2}{*}{$373 \pm 43$} & \multirow{2}{*}{ 506-315 } & \multirow{2}{*}{427} & \multirow{2}{*}{ AA 99102} \\
\hline & & (metapodio) & & & & \\
\hline $\begin{array}{l}\text { A1. Nivel } 2 \\
(20-30 \mathrm{~cm})\end{array}$ & II & gramíneas (camada) & $1416 \pm 37$ & $1379-1285$ & 1322 & AA 85721 \\
\hline \multirow{2}{*}{$\begin{array}{l}\text { A1. Nivel } 3 \\
(30-40 \mathrm{~cm})\end{array}$} & \multirow{2}{*}{ III } & Lama guanicoe & \multirow{2}{*}{$1590 \pm 46$} & \multirow{2}{*}{$1569-1375$} & \multirow{2}{*}{1475} & \multirow{2}{*}{ AA 99103} \\
\hline & & (tibia) & & & & \\
\hline \multirow{2}{*}{ C1 } & \multirow{2}{*}{$\begin{array}{c}\text { Estructura } \\
\text { G }\end{array}$} & Senna aphylla & \multirow{2}{*}{$4786 \pm 46$} & \multirow{2}{*}{ 5603-5330 } & \multirow{2}{*}{5517} & \multirow{2}{*}{$\begin{array}{c}\mathrm{AA} \\
102575\end{array}$} \\
\hline & & (ramas) & & & & \\
\hline \multirow{2}{*}{$\begin{array}{l}\text { A1. Nivel } 4 \\
(40-50 \mathrm{~cm})\end{array}$} & \multirow{2}{*}{ IV } & Lama guanicoe & \multirow{2}{*}{$9375 \pm 91$} & \multirow{2}{*}{$11.068-10.277$} & \multirow{2}{*}{10.601} & \multirow{2}{*}{ AA 99104} \\
\hline & & (radioulna) & & & & \\
\hline \multirow{2}{*}{$\begin{array}{l}\text { A1. Nivel } 4 \\
(40-50 \mathrm{~cm})\end{array}$} & \multirow{2}{*}{ IV } & Lama guanicoe & $9205+90$ & $10699-10250$ & 10.483 & AA 09105 \\
\hline & & (húmero) & $9293 \pm 90$ & $10.099-10.250$ & 10.403 & A \\
\hline A1. Nivel 5 & IV & Prosopis sp. & $9402+60$ & $11057-10433$ & 10632 & AA \\
\hline$(50-60 \mathrm{~cm})$ & IV & (semilla) & $9402 \pm 60$ & $11.05 /-10.433$ & 10.633 & 102574 \\
\hline A1. Nivel 5 & IV & carbón & $9521+20$ & 1108210606 & 10872 & 1485718 \\
\hline$(55-57 \mathrm{~cm})$ & IV & (fogón \#2) & 9331 & $11.083-10.696$ & $10.8 / 2$ & AA $85 / 18$ \\
\hline A1. Nivel 5 & IV & Lama guanicoe & $10.155 \pm$ & $12153-11327$ & 11798 & AA 99106 \\
\hline$(50-60 \mathrm{~cm})$ & IV & (metapodio) & 98 & $12.153-11.327$ & 11.790 & $A A S$ \\
\hline $\begin{array}{l}\text { A1. Nivel } 5 \\
(50-60 \mathrm{~cm}) \\
\end{array}$ & IV & $\begin{array}{c}\text { Retanilla patagonica } \\
\text { (fruto) }\end{array}$ & $9261 \pm 66$ & $10.588-10.250$ & 10.436 & AA 99100 \\
\hline A1. Nivel 5 & V & coprolito & $11.841 \pm$ & 45 & 13658 & AA 85720 \\
\hline$(50-60 \mathrm{~cm})$ & V & (megafauna) & 56 & $13.7 / 1-13.545$ & 13.658 & AA $85 / 20$ \\
\hline A1. Nivel 10 & & coprolito & $13.844 \pm$ & & & \\
\hline $\begin{array}{c}(100-110 \\
\mathrm{cm})\end{array}$ & VII & (megafauna) & 75 & $17.035-16.461$ & 16.759 & AA 85722 \\
\hline
\end{tabular}

Tabla 1. Fechados radiocarbónicos del sitio $\mathrm{CH} 1$. Se encuentran resaltados los fechados puntuales sobre material vegetal (publicados en Barberena 2014). 
en Barberena et al. 2018). Además, se integraron plantas de importancia económica de la región, referenciadas en diferentes fuentes escritas (Azar 2008). Se evaluó la presencia de señales tafonómicas como cortes, decorados y marcas de fricción (Llano et al. 2012).

La metodología utilizada para la identificación de la muestra conformada por el cariopse de maíz fue tanto cuali como cuantitativa (Oliszewski y Olivera 2009). Para las variables cuantitativas se relevaron: longitud, latitud y espesor ( $\mathrm{mm})$. Se utilizó un calibre HL $(150 \times 0,02 \mathrm{~mm})$. Las variables cualitativas que se tuvieron en cuenta fueron: forma, color, dureza y composición del endosperma.

Para realizar el análisis general se han utilizado tres parámetros: a) número de individuos, que corresponde a la suma total de restos de un taxón, excluyéndose los fragmentos; b) densidad, utilizado para comparar muestras de volúmenes diferentes (densidad = conteo absoluto/volumen); y c) Índice de Abundancia Relativa (IAR), que permite comparar cambios y similitudes sin sobrevalorar ciertas concentraciones puntuales de individuos (Popper 1988).

Los patrones de procesamiento son clave para evaluar la incorporación antrópica de los macrorestos a la secuencia del sitio. Los resultados son comparados con diseños experimentales (Capparelli y Lema 2011; Llano et al. 2012) y con datos etnohistóricos (Capparelli y Prates 2015). Se prestó

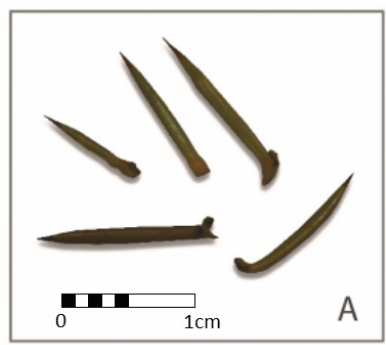

especial atención a las partes del fruto de algarrobo, dada su abundancia. Para la evaluación de las características de los fragmentos botánicos se descartaron las posibilidades de tostado y fragmentación poscarbonización (sensu Capparelli y Prates 2015), ya que los macrorrestos de algarrobo se encontraban en su totalidad secos. Las variables evaluadas fueron el remojo, hervido, molienda para harina $y$ consumo directo.

\section{RESULTADOS}

Se recuperaron 2140 macrorrestos vegetales de las cuadrículas C1 y D1, los cuales han sido identificados en diferentes grados de especificidad (Figura 2). En la
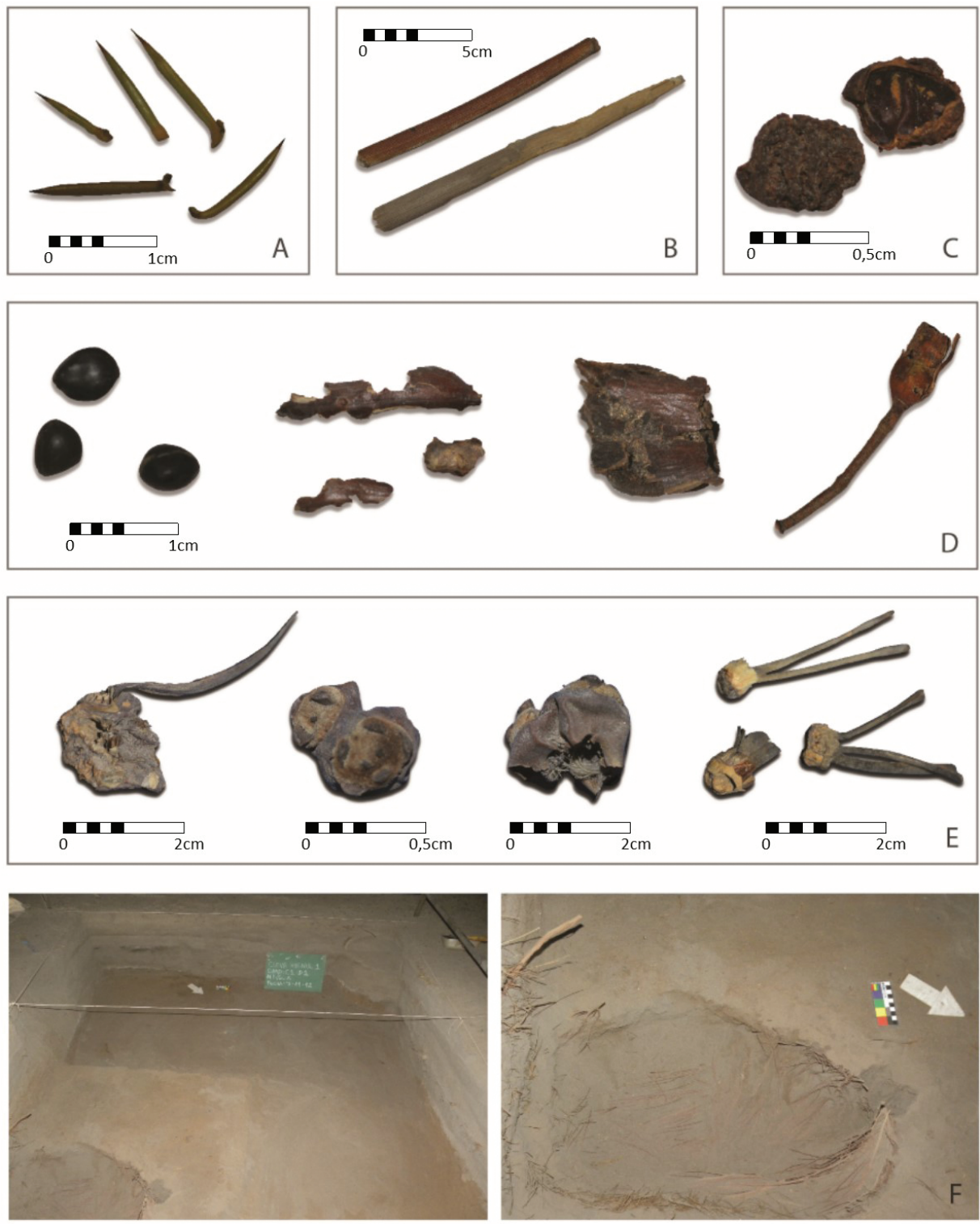

Figura 2. Registro fotográfico de los macrorrestos vegetales recuperados en $\mathrm{CH} 1$. A. Espinas de Berberis grevillana; B. tallos de Equisetum spp. y madera indeterminada; C. Endocarpo de Schinus sp.; D. semillas y epicarpio de Prosopis spp.; E. artejos, espinas y areolas de Maihueniopsis darwinii; F. estructura G de Senna aphylla. 
Tabla 2 se presentan las frecuencias absolutas y relativas por nivel estratigráfico de cada componente, donde se visualiza que de la cuadrícula C1 se recuperaron 606 macrorrestos (28\% del total), mientras que de D1 se recuperaron 1534 especímenes (71\%). Al tratar ambas cuadrículas en forma conjunta por componente cronoestratigráfico, se observa que en el componente 1, que corresponde al Pleistoceno final, se recuperó el $1,6 \%(n=36)$ del total del material arqueobotánico (Tabla 3), entre el que se destaca Prosopis spp. ( $\mathrm{n}=28)$, acompañado por Maihueniopsis darwinii $(\mathrm{n}=6)$, Larrea divaricata $(\mathrm{n}=1)$ y Equisetum spp. $(\mathrm{n}=1)$.

En el componente 2 (Holoceno temprano) se registra el 40,2\% $(n=862)$ de los especímenes vegetales. En la cuadrícula C1 se registraron dos taxa: Prosopis spp. $(\mathrm{n}=39)$ y Schinus polygamus $(\mathrm{n}=2)$, mientras que en D1 la abundancia de Prosopis spp. $(\mathrm{n}=800)$ supera la de todos los periodos evaluados. Un conjunto de taxones se repite en este lapso comparado con el anterior, aunque en baja frecuencia: Maihueniopsis darwinii $(\mathrm{n}=2)$, Larrea divaricata $(n=4)$, Equisetum spp. $(\mathrm{n}=10)$, Schinus sp. ( $\mathrm{n}=2)$ y Larrea nitida $(n=2)$. El componente 3 (Holoceno medio) solo se encuentra representado por una estructura antrópica denominada G, que es un pozo relleno por Senna aphylla, con contenido de endocarpos de Prosopis spp. $(\mathrm{n}=19)$.

En el componente 4 (Holoceno tardío) se evidencia una mayor abundancia en términos de cantidad absoluta de recursos vegetales $(57,08 \%, \mathrm{n}=1221) \mathrm{y}$ una diversificación taxonómica. La densidad de este componente refleja que el
Holoceno tardío es superior a la del resto de los componentes.

Se destaca que la densidad de los macrorrestos de Maihueniopsis en este componente es significativamente superior a la de los componentes previos (Pleistoceno $7,5 \mathrm{ind} / \mathrm{m}^{3}$; Holoceno temprano 3,3 ind $/ \mathrm{m}^{3}$; Holoceno medio 0 ind $/ \mathrm{m}^{3}$; Holoceno tardío 1706,7 ind $/ \mathrm{m}^{3}$ ). Otro caso Ilamativo es el de Prosopis, que, en términos de densidad, es superior en el Holoceno temprano $\left(1398,3 \mathrm{ind} / \mathrm{m}^{3}\right)$ que en el resto de los componentes (Pleistoceno 35,0 ind $/ \mathrm{m}^{3}$; Holoceno medio $950 \mathrm{ind} / \mathrm{m}^{3}$; Holoceno tardío 193,3 ind $/ \mathrm{m}^{3}$ ). Considerando la densidad en

\begin{tabular}{|c|c|c|c|c|c|}
\hline \multicolumn{6}{|c|}{ Sitio arqueológico $\mathrm{CH} 1$-Cuadrícula $\mathrm{C1}$} \\
\hline Componentes & Ext & Taxa & Parte vegetal & $\mathbf{n}$ & $\%$ \\
\hline \multirow{4}{*}{1} & 10 & \multicolumn{4}{|c|}{ S/V } \\
\hline & 9 & \multicolumn{4}{|c|}{ S/V } \\
\hline & 8 & Prosopis spp. & Endocarpo & 1 & $100,00 \%$ \\
\hline & 7 & Prosopis spp. & Endocarpo & 1 & $100,00 \%$ \\
\hline \multirow{4}{*}{ II } & 6 & \multicolumn{4}{|c|}{ S/V } \\
\hline & \multirow{2}{*}{5} & Prosopis spp. & Endocarpo & 17 & $89,50 \%$ \\
\hline & & Schinus polygamus & Endocarpo & 2 & $10,50 \%$ \\
\hline & 4 & Prosopis spp. & Endocarpo & 22 & $100,00 \%$ \\
\hline \multirow{2}{*}{ III } & \multirow{2}{*}{ Estructura G } & Prosopis spp. & Endocarpo & 2 & $66,70 \%$ \\
\hline & & Senna ahylla & Ecofacto & 1 & $33,3 \%$ \\
\hline \multirow{11}{*}{ IV } & \multirow{2}{*}{3} & Maihueniopsis darwinii & Areolas, artejos, espinas & 2 & $33,30 \%$ \\
\hline & & Prosopis spp. & Endocarpo & 4 & $66,70 \%$ \\
\hline & \multirow{5}{*}{2} & Ephedra sp. & Semilla & 1 & $0,20 \%$ \\
\hline & & Larrea sp. & Fruto, hojas & 4 & $0,80 \%$ \\
\hline & & Maihueniopsis darwinii & Areolas, artejos, espinas & 449 & $94,70 \%$ \\
\hline & & Prosopis spp. & Endocarpo & 9 & $1,90 \%$ \\
\hline & & Schinus polygamus & Endocarpo & 11 & $2,30 \%$ \\
\hline & \multirow{4}{*}{1} & Larrea sp. & Fruto, hojas & 1 & $1,30 \%$ \\
\hline & & Maihueniopsis darwinii & Areolas, artejos, espinas & 62 & $77,50 \%$ \\
\hline & & Prosopis spp. & Endocarpo & 16 & $20,00 \%$ \\
\hline & & Xanthium sp. & Involucro & 1 & $1,30 \%$ \\
\hline & & & Subtotal de carporrestos & 606 & \\
\hline \multicolumn{6}{|c|}{ Sitio arqueológico $\mathrm{CH}$ 1-Cuadrícula D1 } \\
\hline \multirow{17}{*}{ I } & \multirow{4}{*}{10} & Equisetum spp. & Tallo & 1 & $2,90 \%$ \\
\hline & & Larrea divaricata & Hoja & 1 & $2,90 \%$ \\
\hline & & Maihueniopsis darwinii & Areolas, artejos, espinas & 6 & $17,60 \%$ \\
\hline & & Prosopis spp. & Endocarpo, semilla & 26 & $76,50 \%$ \\
\hline & 9 & \multicolumn{4}{|c|}{ S/V } \\
\hline & 8 & \multicolumn{4}{|c|}{ S/V } \\
\hline & 7 & \multicolumn{4}{|c|}{ S/V } \\
\hline & 6 & \multicolumn{4}{|c|}{ S/V } \\
\hline & \multirow{3}{*}{5} & Equisetum spp. & Tallo & 6 & $1,10 \%$ \\
\hline & & Prosopis spp. & Endocarpo, semilla & 523 & $98,50 \%$ \\
\hline & & Schinus sp. & Endocarpo & 2 & $0,40 \%$ \\
\hline & \multirow{6}{*}{4} & Equisetum spp. & Tallo & 4 & $1,40 \%$ \\
\hline & & Larrea divaricata & Hoja & 4 & $1,40 \%$ \\
\hline & & Larrea nitida & Hoja & 2 & $0,70 \%$ \\
\hline & & Maihueniopsis darwinii & Areolas, artejos, espinas & 2 & $0,70 \%$ \\
\hline & & Prosopis spp. & Endocarpo, semilla, vaina & 277 & $95,80 \%$ \\
\hline & & Zea mays & Cariopse & 1 & $5,60 \%$ \\
\hline \multirow{2}{*}{ III } & \multirow{2}{*}{ Estructura G } & Prosopis spp. & Endocarpo & 17 & $94,40 \%$ \\
\hline & & Senna ahylla & Ecofacto & 1 & $5,60 \%$ \\
\hline
\end{tabular}

Tabla 2. Continúa en página siguiente. 


\begin{tabular}{|c|c|c|c|c|c|}
\hline \multicolumn{6}{|c|}{ Sitio arqueológico $\mathrm{CH} 1$-Cuadrícula $\mathrm{C} 1$} \\
\hline Componentes & Ext & Taxa & Parte vegetal & $\overline{\mathbf{n}}$ & $\%$ \\
\hline \multirow{17}{*}{ IV } & \multirow{2}{*}{3} & Equisetum spp. & Tallo & 1 & $5,00 \%$ \\
\hline & & Prosopis spp. & Endocarpo & 19 & $95,00 \%$ \\
\hline & \multirow{7}{*}{2} & Berberis grevilleana & Semilla & 1 & $0,20 \%$ \\
\hline & & Equisetum spp. & Tallo & 2 & $0,40 \%$ \\
\hline & & Larrea divaricata & Hoja & 5 & $1,10 \%$ \\
\hline & & Maihueniopsis darwinii & Areolas, artejos, espinas & 414 & $89,00 \%$ \\
\hline & & Prosopis sp. & Endocarpo, semilla, vaina & 40 & $8,60 \%$ \\
\hline & & Schinus sp. & Endocarpo & 1 & $0,20 \%$ \\
\hline & & Xanthium aff. spinosum & Involucro & 2 & $0,40 \%$ \\
\hline & \multirow{8}{*}{1} & Berberis grevilleana & Semilla & 7 & $4,00 \%$ \\
\hline & & Chuquiraga oppositifolia & Receptáculo floral & 2 & $1,10 \%$ \\
\hline & & Equisetum spp. & \begin{tabular}{|l|lll} 
Tallo \\
\end{tabular} & 1 & $0,60 \%$ \\
\hline & & Larrea divaricata & Mericarpo & 36 & $20,50 \%$ \\
\hline & & Maihueniopsis darwinii & Areolas, artejos, espinas & 97 & $55,10 \%$ \\
\hline & & Poaceae & Aff. antecio & 3 & $1,70 \%$ \\
\hline & & Prosopis sp. & Endocarpo & 28 & $15,90 \%$ \\
\hline & & Xanthium aff. cavanillesii & Involucro & 2 & $1,10 \%$ \\
\hline \multirow{2}{*}{\multicolumn{4}{|c|}{$\begin{array}{r}\text { Subtotal de carporrestos } \\
\text { Total de carporrestos } \mathrm{C} 1+\mathrm{D} 1\end{array}$}} & 1534 & \\
\hline & & & & 2140 & \\
\hline
\end{tabular}

Tabla 2. Frecuencias absolutas y relativas por nivel estratigráfico de cada componente de los macrorrestos vegetales recuperados del sitio $\mathrm{CH} 1$-Cuadrículas $\mathrm{C} 1$ y $\mathrm{D} 1$. S/V sin vegetales.

conjunto $(\mathrm{C} 1+\mathrm{D} 1)$ se observan valores superiores en el Holoceno tardío $\left(2035 \mathrm{ind} / \mathrm{m}^{3}\right)$ con respecto al Holoceno temprano $\left(1436,7 \mathrm{ind} / \mathrm{m}^{3}\right)$, Holoceno medio $\left(1050 \mathrm{ind} / \mathrm{m}^{3}\right)$ y el Pleistoceno $\left(45,0 \mathrm{ind} / \mathrm{m}^{3}\right)$ (Tabla 3).

Se registró un cariopse completo de Zea mays (Figura 3) en el nivel 4 de la cuadrícula D1, que estratigráficamente corresponde al componente 2 del Holoceno temprano. Este se encontraba en estado de preservación seco, tiene forma obovada y el color del pericarpio es marrón. Las dimensiones son $7 \mathrm{~mm}$ de longitud por $9 \mathrm{~mm}$ de latitud y $6 \mathrm{~mm}$ de espesor. Se identificó la variedad (sensu Parodi 1959): Zea mays var. amylacea. Los caracteres cualitativos se describen como endosperma totalmente harinoso, blando y blanco, con una porción córnea periférica muy delgada.

Dentro del conjunto de restos de Prosopis predominan los fragmentos de vaina, como artejos enteros y medios artejos (sensu Capparelli y Prates 2015). Se observaron marcas de masticación en los endocarpos enteros $y$, en el caso de endocarpos terminales, se encontraban acompañados de restos de epicarpo en forma de finas hebras entrelazadas, lo que evidencia el consumo fresco sin procesamiento (Figura 2). También se recuperaron epicarpios de consistencia gruesa y fibrosa, signo observado en la experimentación de molienda con vainas secas (Llano et al. 2012).
Por otra parte, algunos de los macrorrestos de Maihueniopsis, madera y la totalidad del material que conforma la estructura G (Senna aphylla) presentaban signos de tinción antrópica por ocre en tonalidades pardo-rojizo.

\section{DISCUSIÓN}

Las escasas reconstrucciones paleoambientales disponibles para la región no registran la existencia de modificaciones sustanciales en la distribución de la vegetación durante el Holoceno (Markgraf et al. 2009; de Porras 2017). Las principales variaciones se relacionan con las fluctuaciones de los límites de las zonas de transición, debido a cambios en la humedad efectiva. Aunque es probable que esto se deba al carácter acotado y fragmentario de las reconstrucciones disponibles, se puede afirmar que la posición del ecotono Monte-Patagonia no se habría corrido lateralmente como para generar un impacto en las comunidades de vegetación que rodean a $\mathrm{CH} 1$, que habrían sido similares a las actuales.

Los restos hallados en el componente 1 -fechado en el Pleistoceno final- se habrían depositado como producto de la ocupación de la cueva por parte de herbívoros. La matriz de estos estratos está conformada en gran parte por material orgánico producto de la desagregación de coprolitos de megafauna. No se cuenta con indicadores contextuales fehacientes que respalden el carácter antrópico de estos materiales (Barberena et al. 2015). En este sentido, se los considera un indicador de las condiciones paleoecológicas en forma previa a la llegada de los primeros grupos humanos a la región.

La secuencia ocupacional de $\mathrm{CH} 1$ indica prácticas de subsistencia de caza y recolección de acuerdo con las cuales el aprovechamiento de los recursos vegetales silvestres, tales como Prosopis, Schinus y Maihueniopsis, se reconocen desde el inicio de las ocupaciones humanas en el Holoceno temprano (componente 2) de A1, C1 y D1. El registro de tallos y semillas, representado por una riqueza de cinco 


\begin{tabular}{|c|c|c|c|c|c|c|c|c|c|c|c|c|c|c|c|c|c|c|}
\hline \multirow{3}{*}{ Taxa } & \multirow{2}{*}{\multicolumn{4}{|c|}{ Pleistoceno (componente 1) }} & \multicolumn{12}{|c|}{ Holoceno } & \multirow{3}{*}{ Totales } & \multirow{3}{*}{ Densidad } \\
\hline & & & & & \multicolumn{2}{|c|}{ Temprano } & \multicolumn{2}{|c|}{ (Componente 2) } & \multicolumn{4}{|c|}{ Medio (Componente 3) } & \multicolumn{4}{|c|}{ Tardío (Componente 4) } & & \\
\hline & C1 & D1 & $\mathrm{A} 1$ & TOTAL & $\mathrm{C} 1$ & D1 & $\mathrm{A} 1$ & TOTAL & C1 & D1 & $\mathrm{A} 1$ & TOTAL & C1 & D1 & $\mathrm{A} 1$ & TOTAL & & \\
\hline Atriplex lampa & - & - & 1 & 1 & - & - & - & $\mathbf{0}$ & - & - & - & $\mathbf{0}$ & - & - & - & $\mathbf{0}$ & 1 & 0,33 \\
\hline Berberis grevilleana & - & - & - & $\mathbf{0}$ & - & - & - & $\mathbf{0}$ & - & - & - & $\mathbf{0}$ & - & 8 & - & 8 & 8 & 4,44 \\
\hline Chuquiraga erinacea & - & - & - & $\mathbf{0}$ & - & - & - & $\mathbf{0}$ & - & - & - & $\mathbf{0}$ & - & - & 1 & 1 & 1 & 0,56 \\
\hline Chuquiraga oppositifolia & - & - & - & $\mathbf{0}$ & - & - & - & $\mathbf{0}$ & - & - & - & $\mathbf{0}$ & - & 2 & - & 2 & 2 & 1,11 \\
\hline Ephedra sp. & - & - & - & $\mathbf{0}$ & - & - & - & $\mathbf{0}$ & - & - & - & $\mathbf{0}$ & 1 & - & - & 1 & 1 & 0,56 \\
\hline Equisetum spp. & - & 1 & - & 1 & - & 10 & - & 10 & - & - & - & $\mathbf{0}$ & - & 4 & - & 4 & 15 & 8,11 \\
\hline Eruca vesicaria & - & - & - & $\mathbf{0}$ & - & - & - & $\mathbf{0}$ & - & - & - & $\mathbf{0}$ & - & - & 1 & 1 & 1 & 0,56 \\
\hline Lagenaria sp. & - & - & - & $\mathbf{0}$ & - & - & - & $\mathbf{0}$ & - & - & - & $\mathbf{0}$ & - & - & 3 & 3 & 3 & 1,67 \\
\hline Larrea divaricata & - & 1 & - & 1 & - & 4 & - & 4 & - & - & - & $\mathbf{0}$ & - & 41 & 8 & 49 & 54 & 29,78 \\
\hline Larrea nitida & - & - & - & $\mathbf{0}$ & - & 2 & - & 2 & - & - & - & 0 & - & - & - & $\mathbf{0}$ & 2 & 1,11 \\
\hline Larrea sp. & - & - & - & $\mathbf{0}$ & - & - & - & $\mathbf{0}$ & - & - & - & $\mathbf{0}$ & 5 & - & - & 5 & 5 & 2,78 \\
\hline Maihueniopsis darwinii & - & 6 & - & 6 & - & 2 & - & 2 & - & - & - & $\mathbf{0}$ & 513 & 511 & 10 & 1034 & 1042 & 577,56 \\
\hline Poaceae & - & - & - & $\mathbf{0}$ & - & - & - & $\mathbf{0}$ & - & - & - & $\mathbf{0}$ & - & 3 & - & 3 & 3 & 1,67 \\
\hline Prosopis spp. & 2 & 26 & 9 & 37 & 39 & 800 & 135 & 974 & 2 & 17 & - & 19 & 29 & 87 & 130 & 246 & 1276 & 769,28 \\
\hline Retanilla patagonica & - & - & 1 & 1 & - & - & - & $\mathbf{0}$ & - & - & - & $\mathbf{0}$ & - & - & - & $\mathbf{0}$ & 1 & 0,33 \\
\hline Schinus polygamus & - & - & - & $\mathbf{0}$ & 2 & - & - & 2 & - & - & - & $\mathbf{0}$ & 11 & - & 16 & 27 & 29 & 16,11 \\
\hline Schinus sp. & - & - & - & $\mathbf{0}$ & - & 2 & - & 2 & - & - & - & $\mathbf{0}$ & - & 1 & - & 1 & 3 & 1,67 \\
\hline Senna ahylla & - & - & - & $\mathbf{0}$ & - & - & - & $\mathbf{0}$ & 1 & 1 & - & 2 & - & - & - & $\mathbf{0}$ & 2 & 8,33 \\
\hline Xanthium aff. cavanillesii & - & - & - & $\mathbf{0}$ & - & - & - & $\mathbf{0}$ & - & - & - & $\mathbf{0}$ & - & 2 & - & 2 & 2 & 1,11 \\
\hline Xanthium aff. spinosum & - & - & - & $\mathbf{0}$ & - & - & - & 0 & - & - & - & 0 & - & 2 & - & 2 & 2 & 1,11 \\
\hline Xanthium sp. & - & - & - & $\mathbf{0}$ & - & - & - & $\mathbf{0}$ & - & - & - & 0 & 1 & - & - & 1 & 1 & 0,56 \\
\hline Zea mays & - & - & - & 0 & - & 1 & - & 1 & - & - & - & 0 & - & - & - & o & 1 & 0,56 \\
\hline \begin{tabular}{|l|} 
Total \\
\end{tabular} & 2 & 34 & 11 & 47 & 41 & 821 & 135 & 997 & 3 & 18 & 0 & 21 & 560 & 661 & 169 & 1390 & 2455 & \begin{tabular}{|l|l}
1429,28 \\
\end{tabular} \\
\hline Densidad & & & 7,9 & 15,7 & & 6,7 & 225 & 553,9 & 10 & 50 & 0 & 87,5 & 20 & 35 & 281,7 & 772,2 & 358,9 & \\
\hline
\end{tabular}

Tabla 3. Distribución y densidad de macrorrestos por componente cronológico.

taxones en este lapso, indica la presencia de plantas arbustivas típicas de monte (i.e., Prosopis) y plantas indicadoras de zonas húmedas (i.e., Equisetum), que fructifican en época estival, lo que sugiere el uso de laderas cerca de fuentes de agua y ambientes húmedos, los cuales habrían estado presentes en
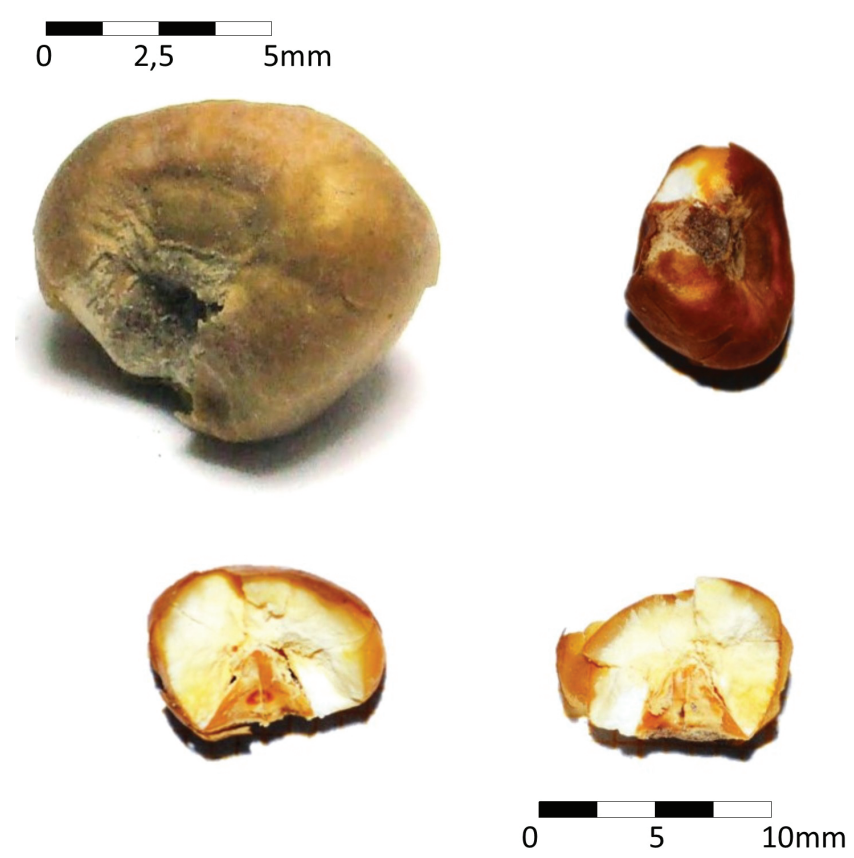

Figura 3. Macrorresto de Zea mays var. Amylacea. forma discontinua en el entorno de $\mathrm{CH} 1$. El registro del componente 2 indica el consumo redundante de algarrobo (Prosopis spp.) con fecha taxón directa para una muestra de frutos del nivel 5 de la cuadrícula A1 con una mediana calibrada de 10.633 años AP (Tabla 1).

Los componentes 2, 3 y 4 evidencian señales de procesamiento y depositación antrópica de estos restos, ya que los endocarpos se encuentran fragmentados y con señales de fricción (Figura 2). Esto sugiere que, al menos, una parte de estas plantas fueron procesadas antes de ser utilizadas. Además del uso alimentario, los macrorrestos presentaron impregnaciones con pigmento colorante en Senna aphyIla (componente 3), Maihueniopsis y Equisetum (componente 4). Los macrorrestos asignados a Ephedra, Larrea y Xanthiun no presentaban marcas de procesamiento.

Si bien los usos potenciales de las plantas están limitados por factores biológicos, el comportamiento humano juega un papel importante en la definición del uso de un recurso (Ford 1979). Las evidencias obtenidas en referencia al procesamiento de Prosopis permiten marcar la existencia de diferentes estrategias en el 
Holoceno temprano (componente 2), comparado con el Holoceno tardío (componente 4). En el componente 2 se observa que los restos de algarrobo son producto del consumo directo mediante mascado de vainas sin procesamiento previo bajo la forma de tostado o hervido. Por el contrario, en el componente 4 del Holoceno tardío se observan marcas en las vainas compatibles con un consumo que implicó procesamiento como la molienda. Se señala que, aunque en baja frecuencia, en el sitio $\mathrm{CH} 1$ y en la localidad Huenul en general se registró la presencia de artefactos líticos de molienda.

En cuanto a la estacionalidad de este tipo de recurso, en general se establecieron en períodos de producción de frutos y semillas, los cuales coincidieron con el verano. Al analizar estas tendencias desde el rendimiento nutricional y la fenología vegetal, es esperable que en el pasado las poblaciones hayan consumido mayor cantidad de algarrobo, ya que no solo está ranqueado en primer lugar por su rendimiento calórico (Llano y Ugan 2010), sino que tiene una amplia época de fructificación, disponibilidad para el consumo y posibilidad de almacenamiento. En el caso de Maihueniopsis, la época de fructificación está acotada a los meses de noviembre a enero (Roig 1998), que sería un indicador de uso estival. En tanto que el uso tecnológico para el acondicionamiento del lugar (Senna aphylla) es potencialmente anual.

La interpretación del consumo de especies que ofrece el entorno se corresponde con los estudios de ecología isotópica regional para el extremo norte de la provincia del Neuquén (Gordón et al. 2017), ya que todos los ecosistemas tendrían una señal dominante de base $C_{3}$. Este dato se sustenta en la señal isotópica de plantas de esa vía fotosintética registrada en el muestreo vegetacional en la localidad Huenul, así como en los valores de ${ }^{13} \mathrm{C} /{ }^{12} \mathrm{C}$ registrados para muestras de Lama guanicoe de $\mathrm{CH} 1$ (Barberena et al. 2018).

En base al pequeño conjunto de $\mathrm{CH} 1$ que es asignado a esta instancia temprana del poblamiento humano regional, se registra el consumo de algarrobo y guanaco (Barberena et al. 2015), que son respectivamente los taxones de más alto ranking dentro de los recursos vegetales y animales en la región (Llano 2015). El único ejemplar de Zea mays se recuperó en el nivel 4 asignado al componente 2 . Se considera altamente probable su migración vertical, sobre la base de los registros disponibles para este taxón exclusivamente para el Holoceno tardío en zonas cercanas a la región de estudio (Pérez y Erra 2011; Lema et al. 2012), como así también en la vertiente occidental de la cordillera (Roa et al. 2018). Se está preparando un informe que permita posicionarlo a nivel cronológico y morfológico (Llano en preparación).

El componente 3 del Holoceno medio no resulta comparable a los otros, ya que corresponde exclusivamente a la estructura $G$, que es un pozo relleno de material vegetal teñido con pigmento rojo (fecha taxón para Senna aphylla con una mediana calibrada de 5517 años AP). Esta es la única evidencia registrada en estratigrafía para este período de tiempo escasamente representado a nivel arqueológico macrorregional (Neme y Gil 2009; Barberena et al. 2015). Esta estructura contiene también escasos especímenes de algarrobo. El registro en el Holoceno tardío reflejó una mayor densidad y riqueza especifica de especies recuperadas, con un total de 14 taxones identificados, entre los que es dominante Maihueniopsis darwinii. Este conjunto arqueobotánico no solo es abundante, sino que también indica el procesamiento de la planta in situ con trazas de pigmento rojo en ciertos casos.

La disminución en la densidad de algarrobo en el Holoceno tardío comparado con el Holoceno temprano refleja una conducta que tiene correlato con los relevamientos actuales en torno al sitio (Sosa 2016) y con los estudios macrobotánicos y palinológicos realizados en paleomadrigueras de roedores de la localidad Huenul (Llano et al. 2019) ya que se interpreta que, entre 12.000 y 8000 AP, el entorno ofrecía una mayor abundancia de algarrobo. La disponibilidad hídrica también podría condicionar el patrón de distribución espacial de los algarrobales (Villagra et al. 2004), que se localizan en sitios donde se disponga de agua freática anual. Actualmente se ha observado la amplia disponibilidad de este taxón en el entorno del arroyo Buta Có, ubicado a ca. $4 \mathrm{~km}$ de $\mathrm{CH} 1$, donde está situada la localidad arqueológica Puesto Cuello-Cueva Yagui.

Por último, este registro posiblemente evidencie la recolección cuando los ocupantes del sitio realizaban movimientos con objetivos diversos. En este sentido, por su disponibilidad dispersa en la región, una posibilidad es que la recolección de plantas ocurriera en forma complementaria en el marco de la realización de otras actividades (Binford 1979), tales como búsqueda de agua, que no está disponible en forma permanente en el entorno de $\mathrm{CH} 1$, recursos líticos, o presas de caza como el 
guanaco. Los frutos y semillas de las plantas fueron probablemente consumidos siempre que estuvieron disponibles, lo que evidencia así la especificidad en las zonas de recolección, pero también que tenían suficiente importancia para ser llevados al sitio, donde se habrían realizado las actividades de procesamiento $y$, muy posiblemente, de consumo.

\section{CONCLUSIÓN}

El registro arqueobotánico presenta variaciones en la densidad y riqueza específica de plantas entre las cuadrículas analizadas. Los restos estudiados sugieren una mayor intensidad de descarte de vegetales en el componente 4, que representa entre 1500-300 años AP. El consumo y uso de plantas habría aumentado con el tiempo, representado por una gran riqueza del registro de semillas, especialmente cuando se comparan las etapas iniciales de ocupación con las tardías. Sin embargo, cabe señalar que, dado el carácter breve de las ocupaciones iniciales en $\mathrm{CH} 1$, la muestra puede ser muy poco representativa de la dieta humana. Sí se destaca el registro de formas de procesamiento más intensivas en el Holoceno tardío.

La abundancia de algarrobo sustenta su rol como alimento, basado principalmente en la ingesta de proteínas y lípidos (Llano y Ugan 2010), lo que se corresponde con la categorización de alimento suplementario propuesto por Tanaka (1998). Las marcas de procesamiento en los macrorrestos indican un consumo en fresco en momentos tempranos, que puede ser caracterizado como "expeditivo", mientras que, hacia los períodos tardíos, el registro muestra un aprovechamiento más intensivo del recurso, en asociación a una mayor inversión de trabajo, que permite aumentar el rendimiento calórico.

Se debe destacar que la presencia del cariopse de maíz constituye hasta el momento el único macrorresto del taxón en el noroeste de Patagonia, si bien se cuenta con antecedentes de esta especie representada por microrrestos (Pérez y Erra 2011; Lema et al. 2012). El marco regional de evidencias no sugiere la producción local de esta especie doméstica, sino el ingreso a la región y al sitio bajo mecanismos indirectos tales como el intercambio (Gil 2006). En este sentido, esta evidencia puede ser agrupada con los especímenes de Lagenaria sp. recuperados en $\mathrm{CH} 1$, algunos de los cuales presentan evidencias de decoración mediante la técnica de pirograbado (Llano y Barberena 2013).
El registro arqueobotánico ofrece un marco para situar los análisis de amplitud de dieta y pautas de movilidad humana para la recolección y explotación de recursos en diferentes épocas del año. Los resultados presentados reflejan la conducta de trasladar estas plantas al sitio, posiblemente en forma complementaria al consumo, pero también puede ser que su aprovisionamiento se haya realizado para cubrir otras necesidades; es factible el ingreso accidental de la planta recolectada y que la parte realmente usada corresponda a otras, como las cactáceas pigmentadas o las maderas. Este punto es un tema pendiente en la agenda de análisis botánico del sitio, puesto que se recuperaron maderas con pirograbados similares a los dibujos en el epicarpio de Lagenaria sp. (Llano y Barberena 2013). Actualmente, la representación de motivos en soportes de material leñoso está siendo analizada como parte de un trabajo doctoral que involucra el estudio sobre la comunicación visual mediante múltiples tipos de imágenes presentes en el registro arqueológico del área y en diferentes tipos de soporte (Romero Villanueva y Barberena 2017). La arqueobotánica permite generar una imagen más completa de las sociedades humanas que habitaron el noroeste de Patagonia.

\section{Agradecimientos}

Las investigaciones son financiadas por CONICET y AGENCIA (Proyectos PIP 0301, PICT 2014-0940, PICT 201-0062). Agradecemos a la Subsecretaría de Cultura de la Provincia del Neuquén, particularmente a Claudia Della Negra y Pablo Azar. A las Dras. Verónica Lema y Nurit Oliszewski por el intercambio de ideas. Nuestro trabajo ha sido posible gracias al apoyo de las comunidades de Barrancas y Buta Ranquil, por lo que agradecemos a Juvenal Urrutia, Paulina Valenzuela y Hugo Zalazar. Finalmente, agradecemos a los integrantes del proyecto de investigación por todo su apoyo y a la Lic. Mariana Martínez por el diseño de las imágenes.

\section{REFERENCIAS CITADAS}

Azar, P.

\footnotetext{
2008 Utilización de vegetales en las sociedades indígenas norpatagónicas (Neuquén y Río Negro). Contribución para una base de datos. En Rastros: Arqueología e Historia de la cuenca del río Limay, compilado por M. Fernández. Serie monográfica, Volumen 2. CD Rom. Buenos Aires.
} 
Barberena, R.

2014 Discordancias y discontinuidades en Patagonia septentrional: Crono-estratigrafía de Cueva Huenul 1 (Neuquén, Argentina). En Arqueología en ambientes de altura en Mendoza y San Juan, editado por V. Cortegoso, V. Durán y A. Gasco, pp. 203-219. EDIUNC, Mendoza.

Barberena, R., K. Borrazzo, A. Rughini, G. Romero, M. P. Pompei, C. Llano, M. E. de Porras, V. A. Durán, C. Stern, A. Re, D. Estrella, A. Forasiepi, F. J. Fernández, M. Chidiak, L. Acuña, A. Gasco y M. N. Quiroga

2015 Perspectivas arqueológicas para Patagonia Septentrional: Sitio Cueva Huenul 1 (Provincia del Neuquén, Argentina). Magallania 43 (1): 137-163.

Barberena, R., A. Tessone, M. N. Quiroga, F. Gordón, C. Llano, A. Gasco, J. Paiva y A. Ugan

2018 Guanacos y ecología isotópica en el norte del Neuquén: El registro de Cueva Huenul 1. Revista del Museo de Antropología 11 (1): 7-14.

Belmar, C., C. Méndez y O. Reyes

2017 Hunter-gatherer plant resource use during the Holocene in central western Patagonia (Aisén, Chile, South America). Vegetation History and Archaeobotany 26 (6): 607-625.

Binford, L.

1979 Organization and formation processes: looking at curated technologies. Journal of Archaeological Research 35 (3): 255-273.

Capparelli, A. y V. Lema

2011 Recognition of post-harvest processing of algarrobo (Prosopis spp.) as food from two sites of Northwestern Argentina: an ethnobotanical and experimental approach for desiccated macroremains. Archaeological and Anthropological Sciences 3 (1): 71-92.

Capparelli, A. y L. Prates

2015 Explotación de frutos de algarrobo (Prosopis spp.) por grupos cazadores recolectores del noreste de Patagonia. Chungara 47 (4): 549-563.

Caruso, L.

2012 Modalidad de adquisición y usos de los recursos leñosos entre grupos cazadores-recolectores patagónicos (Argentina): metodología y técnicas de estudio de material leñosos arqueológico. Tesis Doctoral inédita. Universitat Autónoma de Barcelona, Barcelona.

Ciampagna, M. L. y E. Mange

2017 Primeros estudios arqueobotánicos del sitio Cueva Epullán chica (depto. Collón Curá, provincia del Neuquén). En Anti. Latinoamérica: una mirada desde el presente hacia el pasado, editado por M. T. Haro, A. M, Rocchietti, M. A. Runcio, M. V. Fernández y O. Hernández de Lara, pp. 167-183. Aspha, Ciudad Autónoma de Buenos Aires.

Cordero, R., S Lanzelotti y H. Panarello 2002 INGEIS Radiocarbon Laboratory Dates IV Radiocarbon 44: 181-193.

Crivelli Montero, E., U. F. J. Pardiñas y M. Fernández 1996 Introducción, procesamiento y almacenamiento de macro vegetales en la Cueva Epullán Grande (Provincia del Neuquén). En Arqueología, solo Patagonia,

Ponencias de la Segundas Jornadas de Arqueología de Patagonia, editado por J. Gómez Otero, pp. 49-59.

Centro Nacional Patagónico (CENPAT), Puerto Madryn.

de Porras, M. E.

2017 Escenarios paleoambientales y paleoclimáticos de la Patagonia norte (Neuquén) desde el tardiglacial. En El poblamiento humano del norte de Neuquén. Estado actual del conocimiento y perspectivas, editado por $\mathrm{F}$. Gordón, R. Barberena y V. Bernal, pp. 23-33. Aspha, Ciudad Autónoma de Buenos Aires.

Fernández, J.

1974-1975. Estudios sobre el arte rupestre de la provincia del Neuquén. Anales de Arqueología y Etnología 29-31: 5-36.

Fernández, F. J., P. Teta, R. Barberena y U. F. J. Pardiñas 2012 Small mammal remains from Cueva Huenul 1, northern Patagonia, Argentina. Taphonomy and paleoenvironments since the Late Pleistocene. Quaternary International 278: 22-31.

Ford, R.

1979 Paleoethnobotany in American Archaeology. En Advances in Archaeological Method and Theory, t. 2, editado por M. Schiffer, pp. 285-336. Academic Press, Nueva York.

Gil, A. F.

2006 Arqueología de La Payunia (Mendoza, Argentina): El poblamiento humano en los márgenes de la agricultura. BAR Series 1477. Archaeopress, Oxford.

Gordón, F., S. I. Perez, A. Hajduk, M. Lezcano y V. Bernal 2017 Dietary patterns in human populations from Northwest Patagonia during Holocene: an approach using Binford's frames of reference and Bayesian isotope mixing models Archaeological and Anthropological Sciences 10 (6): 1347-1358. doi 10.1007/s12520-016-0459-0 
Hogg, A. G., Q. Hua, P. Blackwell, M. Niu, C. Buck, T. Guilderson, T. Heaton, J. Palmer, P. Reimer, R. Reimer, C.

Turney y S. Zimmerman

2013 SHCal13 Southern Hemisphere calibration, 0-50,000 years cal BP. Radiocarbon 55 (4): 1889-1903.

Lema, V, C. Della Negra y V. Bernal 2012 Explotación de recursos vegetales silvestres y domesticados en Neuquén: implicancias del hallazgo de restos de maíz y algarrobo en artefactos de molienda del Holoceno tardío. Magallania 40: 229-247.

Llano, C.

2014 The exploitation of plant resources by huntergatherer societies in southern Mendoza, Argentina. Darwiniana 2 (1): 96-111.

2015 On optimal use of a patchy environment: archaeobotany in the Argentinean Andes (Argentina). Journal of Archaeological Science 54: 182-192.

Llano, C. y R. Barberena 2013 Explotación humana de especies vegetales en Patagonia septentrional: el registro arqueobotánico de Cueva Huenul 1 (Provincia de Neuquén, Argentina). Darwiniana 1 (1): 5-19.

Llano, C. y A. Ugan

2010 Rendimiento Económico de plantas silvestres del sur de Mendoza: valores nutricionales, costos de manejo e interpretación del registro arqueológico. En Etnobotánica, tradiciones y transformaciones, editado por M. L. Pochettino, A. Ladio y P. Arenas, pp. 44-48. CYTED-Red Iberoamericana de Saberes y Prácticas Locales sobre el Entorno Vegetal (RISAPRET).

Llano, C., M. E. de Porras, O. Beltrame y R. Barberena 2019 Holocene paleoenvironments and human occupations in the western limit of the South American Arid Diagonal (northwestern Patagonia, Argentina). The Holocene. MS.

Llano, C., A. Ugan, A. Guerci y C. Otaola 2012 Arqueología experimental y valoración nutricional del fruto de algarrobo (Prosopis flexuosa): inferencias sobre la presencia de macrorrestos en sitios arqueológicos. Intersecciones en Antropología 13 (2): 513-524.

Markgraf, V., C. Whitlock, R. S. Anderson y A. García 2009 Late Quaternary vegetation and fire history in the northernmost Nothofagus forest region: Mallín Vaca Lauquen, Neuquén Province, Argentina. Journal of Quaternary Science 24 (3): 248-258.

Mendía, J. M. 2006 Aptitud de las tierras de Neuquén para el pastoreo. Instituto Nacional de Tecnología Agropecuaria (INTA),
Buenos Aires.

Mostacedo, B. y T. Fredericksen

2000. Manual de métodos básicos de muestreo y análisis en ecología vegetal. Proyecto de Manejo Forestal Sostenible (BOLFOR), Santa Cruz, Bolivia.

Neme, G. y A. Gil

2009 Human occupation and increasing mid-Holocene aridity: southern Andean perspectives. Current Anthropology 50 (1): 149-163.

Oliszewski, N. y D. E. Olivera

2009 Variabilidad racial de macrorrestos arqueológicos de Zea mays (Poaceae) y sus relaciones con el proceso agropastoril en la Puna Meridional Argentina (Antofagasta de la Sierra, Catamarca). Darwiniana 47 (1): 77-91.

Páez, M., F. Quintana y C. Pérez 2004 Biogeografía de las Regiones Áridas y Semiáridas entre los $35^{\circ}-39^{\circ}$ S, Argentina. Boletín de la Sociedad Argentina de Botánica 39 (3- 4): 171-180.

Parodi, L.

1959. Enciclopedia Argentina de Agricultura y Jardinería 1. ACME, Buenos Aires.

Pérez de Micou, C.

1991 Fuego, fogones y señales. Una aproximación etnoarqueológica a las estructuras de combustión en el Chubut medio. Arqueología 1: 125-150.

Pérez, A. E. y G. Erra

2011 Identificación de maíz de vasijas recuperadas de la Patagonia noroccidental argentina. Magallania 39 (2): 309-316.

Pompei, M. P., R. Barberena, M. E. de Porras, K. Borrazzo, A. Rughin y A. Gil

2012 Late Quaternary Ecosystems and Humans in Northern Patagonia (Neuquén, Argentina). Southbound. Late Pleistocene Peopling of Latin America. Current Research in the Pleistocene: 187-190.

Popper, V. S.

1988 Selecting Quantitative Measurements in Paleoethnobotany. En Current Paleoethnobotany. Analytical Methods and Cultural Interpretations of Archaeological Plant Remains, editado por C. A. Hastorf y V. S. Popper, pp. 53-71. University of Chicago Press, Chicago.

Roa, C., D. Bustos, H. Ramírez y R. Campbell 2018 Entre la Pampa y el Pacífico sur. Evaluando la dispersión más austral de cultígenos en el Cono Sur americano. VII Jornadas Arqueológicas Cuyanas. Malargüe, 9-11 de mayo. 
Roig, F. A.

1998 La vegetación de la Patagonia. Flora patagónica 1: 48-166.

Roig, F. A., S. Roig-Juñent y V. Corbalán

2009. Biogeography of the Monte Desert. Journal of Arid Environments 73: 164-172.

Romero Villanueva, G. y R. Barberena 2017. Los huesos de guanaco pintados de Cueva Huenul 1 (norte del Neuquén, Patagonia septentrional). Relaciones de la Sociedad Argentina de Antropología XLII (2): 369-377.

Schobinger, J.

1985. Áreas intermedias o marginales. En Cazadores de la Patagonia y agricultores andinos. Arte rupestre de la Argentina, editado por J. Schobinger y C. J. Gradin, pp. 80-91. Encuentro, Madrid.

Sosa, $\mathrm{P}$.

2016 Descifrando interacciones de cazadoresrecolectores con los recursos vegetales silvestres a lo largo del Holoceno: Arqueobotánica del sitio arqueológico Cueva Huenul 1 (Neuquén, Argentina). Tesis de Licenciatura inédita. Facultad de Ciencias Exactas y Naturales, Universidad Nacional de Cuyo, Mendoza.
Tanaka J.

1998 Subsistence ecology of central Kalahari San. En Hunter-gatherers: studies of the !Kung San and their neighbors, editado por R. Lee e I. DeVore, pp. 98-119. Harvard University Press, Cambridge.

Tietze, E., Barberena, R. y O. Beltrame 2019 Paleoparasite assemblages from feline coprolites from the Pleistocene-Holocene Transition in Patagonia: Cueva Huenul 1 archaeological site (Argentina). MS.

Villagra, P. E., Cony, M. A., Mantován, N. G., Rossi, B. E., Loyarte, M. G., Villalba, R. y Marone, L.

2004 Ecología y manejo de los algarrobales de la Provincia Fitogeográfica del Monte. Ecología y Manejo de Bosques Nativos de Argentina. Editorial Universidad Nacional de La Plata, La Plata. 
